\title{
NEUTRAL WINDS IN THE LOWER THERMOSPHERE FROM DYNAMICS EXPLORER 2
}

\author{
T.L. Killeen ${ }^{1}$, B. Nardi ${ }^{1}$, P.N. Purcell ${ }^{1}$, R.G. Roble ${ }^{2}$, \\ T.J. Fuller-Roweli ${ }^{3}$, and D. Rees ${ }^{4}$
}

\begin{abstract}
Doppler line profile measurements of the OI $\lambda 557.7 \mathrm{~nm}$ "green line" emission, made by the Fabry-Perot interferometer on Dynamics Explorer 2, have provided altitude profiles of the meridional component of the lowerthermospheric neutral wind. The wind inversion technique of Nardi [1991] has been used to extract the neutral wind profiles from the line-of-sight measurements. Individual $\lambda 557.7 \mathrm{~nm}$ Doppler line profiles and inverted volume-emission-rate and neutral-wind profiles are presented. Neutral wind measurements from the $-120 \mathrm{~km}$ altitude level, obtained on multiple orbital passes over the summer hemisphere polar region, have been merged to produce a synthesized averaged "vector" wind field in geomagnetic coordinates. The wind pattern exhibits a region of anticyclonic vorticity in the daytime sector of the magnetic polar cap. Averaged winds of $\sim 300$ $\mathrm{m} / \mathrm{sec}$ in the equatorial direction are observed in the early morning sector. The satellite winds are in reasonable agreement with the predictions of the NCAR and UCL thermosphere general circulation models, with significant regional discrepancies evident in both magnitude and direction.
\end{abstract}

\section{Introduction}

Observations made from the low-altitude, polar-orbiting, Dynamics Explorer-2 (DE-2) spacecraft during solar-cyclemaximum conditions have shown that magnetosphereionosphere-thermosphere coupling processes profoundly influence the upper-thermospheric neutral wind pattern at high latitudes. Due to ion drag, these winds develop vortex structures that resemble typical F-region ion convection cells. The geometrical characteristics of the upper-thermospheric high-latitude wind pattern and its dependence on local time, season, solar cycle, geomagnetic activity, and interplanetary magnetic field orientation have been the subject of numerous recent experimental studies [e.g., Killeen and Roble, 1988].

The lower thermospheric wind structure is not as well understood, owing to the difficulty of making in-situ observations in a region where spacecraft lifetimes are short and rocket flights infrequent. Measurements of lower thermospheric winds from various ground-based radars, however, have provided extensive data sets for specific installation locations [e.g., Johnson and Luhmann, 1985; Manson et al., 1989; Johnson, 1990]. There have also been

Department of Atmospheric, Oceanic, and Space Sciences, The University of Michigan

2 High Altitude Observatory, National Center for

Atmospheric Research

${ }^{3}$ CIRES University of Colorado/NOAA Space Environment

Laboratory

${ }^{4}$ Atmospheric Physics Laboratory, University College

London

Copyright 1992 by the American Geophysical Union.

Paper number 92GL01023

$0094-8534 / 92 / 92 \mathrm{GL}-01023 \$ 03.00$ several ground-based optical measurements of winds using the lower green line $\lambda 557.7 \mathrm{~nm}$ emission at $\sim 97 \mathrm{~km}$ [Cogger et al., 1985; Wiens et al., 1988; Lloyd et al., 1990] and individual $\mathrm{OH}$ emission lines at $-86 \mathrm{~km}$ [Hernandez and Smith, 1984; Rees et al., 1990]. The ground-based measurements made at auroral latitudes have indicated that the dynamical structure is controlled by upward-propagating tides and gravity waves [e.g., Manson et al., 1988], and that magnetospheric effects are also significant at times [Cogger et al., 1985]. There have been no lower-thermospheric wind measurements from deep within the geomagnetic polar cap.

The DE-2 Fabry-Perot interferometer, FPI [Hays et al., 1981], was designed to measure primarily the meridional component of the upper-thermospheric neutral wind using the OI $\lambda 630.0 \mathrm{~nm}$ emission, peaking at $\sim 240 \mathrm{~km}$. In order to measure wind profiles at lower altitudes, however, a $557.7 \mathrm{~nm}$ filter was also used routinely in a background, time-shared mode and a large data base of many hundreds of orbits exists. These data have now been reduced to provide detailed Doppler-line-profile measurements of the daytime, twilight, and auroral OI $\lambda 557.7 \mathrm{~nm}$ emissions. The Doppler line profiles, in turn, have been inverted to provide volume emission rate (VER) and meridional wind altitude profiles.

We present our first results of the DE-2 lowerthermospheric meridional wind measurements and compare the binned and averaged data from the high-latitude $120 \mathrm{~km}$ altitude region with the winds calculated by two thermosphere general circulation models (TGCMs). The instrumental technique and experimental data are first presented, followed by model comparisons and, lastly, a summary of conclusions.

\section{Neurral Wind Observations}

The DE-2 FPI was a plane-etalon interferometer, with a $1.26 \mathrm{~cm}$ etalon gap, a $3.5 \mathrm{~cm}$ diameter aperture, an 8-position filter wheel, a 12-channel photon-counting detector, and horizon-scanning optics. The radius of the roughly conical FPI field of view (at the tangent point to the Earth's limb) was in the range $7-25 \mathrm{~km}$, depending on the zenith angle of the view direction. The Doppler-line-profile analysis technique used has been previously described by Killeen and Hays [1984].

Figure 1 shows individual dayside $\lambda 557.7 \mathrm{~nm}$ spectrograms from three different tangent-point altitudes, obtained during orbit 7193. Each 12-channel spectrogram shows the instrumental counts accumulated in $0.88 \mathrm{~s}$. The free spectral range of the FPI at $557.7 \mathrm{~nm}$ is $0.0123 \mathrm{~nm}$ and the wavelength interval corresponding to an individual channel width is $0.0014 \mathrm{~nm}$. Successive peaks, corresponding to different integral orders of interference, are separated by 8.85 channels. The $557.7 \mathrm{~nm}$ peak appears in channel 8 , with a portion of the adjacent order appearing in channels 1 and 2 . The width of the peak is governed by the ambient temperature.

Figure 1c shows the spectrogram obtained for a tangent point altitude of $82 \mathrm{~km}$. At and below these altitudes on the dayside of the orbit, Rayleigh-scattered sunlight contaminates the Doppler line profile, as is evident by the characteristic 


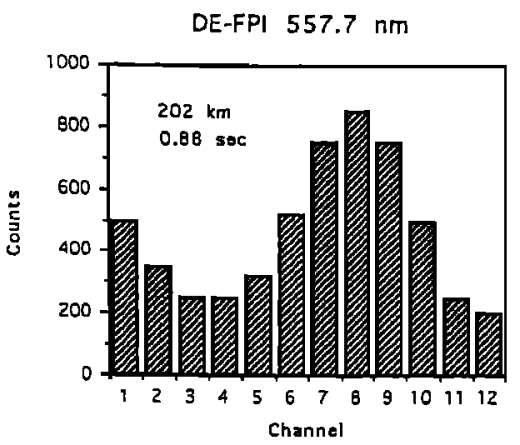

a)

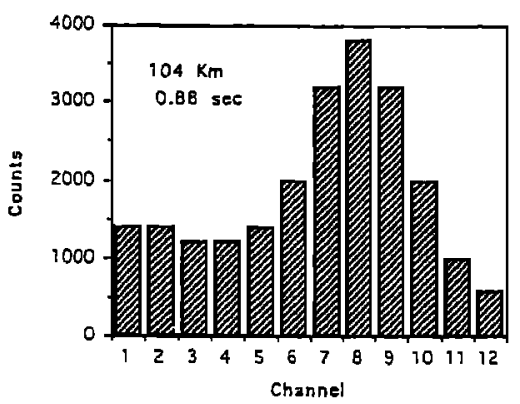

b)

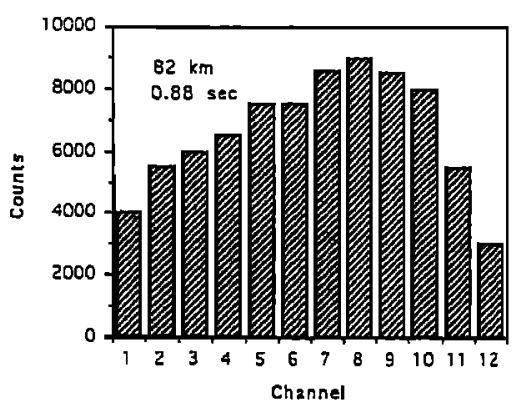

c)

Fig. 1. Examples of 12-channel FPI spectrograms obtained on the dayside of DE-2 orbit 7193 .

"wedge" shape of the spectrogram. The FPI provides uncontaminated daytime Doppler measurements down through the altitude of the lower green line emission at $\sim 97 \mathrm{~km}$. Such low-altitude daytime measurements of green line emissions have never before been available and are possible here only because of the high-spectral resolution of the FPI.

Many thousands of such spectra from hundreds of orbital passes have been analyzed to provide wind and VER data. Figure 2 shows a typical daytime surface brightness profile and the corresponding inverted VER profile for a single FPI limb scan. The VER profile was derived using the inversion technique of Nardi [1991]. Briefly, this technique is based on a constrained "onion-peel" matrix inversion. The constraint used in the inversion involves the assumption of a multipleChapman-layer form for the VER profile. The data points denoted by rectangles in Figure 2a represent the line-of-sight brightness measurements and the crosses represent the calculated (or reconstituted) brightnesses that are consistent with the inverted VER profile of Figure $2 \mathrm{~b}$. The close agreement between the measured and reconstituted profiles in Figure 2a indicates that the derived VER profile is consistent with the experimental measurements. It can be seen that the daytime brightness profile (Figure $2 a$ ) increases monotonically with decreasing altitude, and the VER profile (Figure 2b) depicts two broad peaks, corresponding to the lower green line emission near $97 \mathrm{~km}$ and the higher altitude emission layer

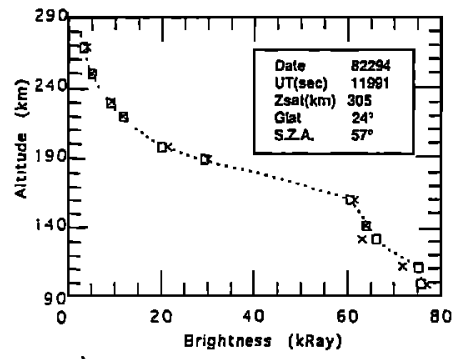

a)

Fig. 2. (a) Measured surface brightness for a single limb scan of the DE-2 FPI viewing the $\lambda 557.7 \mathrm{~nm}$ emission. The boxes represent the direct measurements and the crosses represent the calculated brightnesses based on the derived VER profile. (b) Derived VER profile. The experimental errors are smaller than or commensurate with the size of the plot symbols.

peaking near $170 \mathrm{~km}$ altitude. These results are consistent with the sounding rocket data of Wallace and McElroy [1966].

Figure 3 shows three examples of neutral wind profiles derived from the $\lambda 557.7 \mathrm{~nm}$ emission Doppler line profiles, using the wind inversion technique of Nardi [1991]. The wind inversion technique is considerably more complicated than that for VERs. Briefly, it involves developing a system of equations which characterize the measurement and include explicit terms for the effects of i) instrumental spectral broadening, ii) weighting by local VERs, iii) field-of-view blurring due to optical aberrations and viewing geometries, and iv) variations in brightness within the field of view. The equations are linearized using a Taylor-series expansion about the apparent (or line-of-sight) winds and solved using a singular-value-decomposition method.

The profiles shown in Figure 3 are for daytime low-latiude conditions, with positive winds representing the northward direction. The error bars incorporate uncertainties due to counting statistics and the wind inversion technique. Owing to the large conical field of view $(-20 \mathrm{~km}$ radius at the lowest altitudes) and the relatively long horizontal path lengths through the emitting atmosphere along the line of sight $(-200$ $600 \mathrm{~km}$ ), the remote FPI measurements tend to smear out large spatial shear structures seen in published rocket profiles [Heppner and Miller, 1982]. These and many other DE-2 wind profiles, however, do depict wavelike structures in the vertical with amplitudes that increase in magnitude with altitude, as would be expected from gravity waves propagating vertically. The wind magnitudes are generally $<100 \mathrm{~m} / \mathrm{sec}$, with error bars of $\sim \pm 7 \mathrm{~m} / \mathrm{sec}$ at the lowest altitudes and $\sim \pm 50 \mathrm{~m} / \mathrm{sec}$ at altitudes $>250 \mathrm{~km}$ where the emission rates drop to low magnitudes.

\section{Averaged Data-Model Comparisons}

Wind measurements from the $\lambda 557.7 \mathrm{~nm}$ observations made at high southern (summer) latitudes for tangent point altitudes between 100 and $140 \mathrm{~km}$ have been binned and averaged in geomagnetic polar coordinates for two separate 40-day periods of the DE mission. The bin dimensions were $10^{\circ}$ latitude and 1 hour local time. The two data-collection periods were centered on November 12, 1982 and on January 27,1983 . The total number of orbital passes used in this study was $\sim 100$.

In the first data collection period, the DE-2 spacecraft crossed the southern hemisphere polar region in the 19:00- 
MERIDIONAL WIND [DE2-FPI]

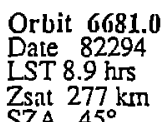

Csat $2775^{\circ} \mathrm{km}$
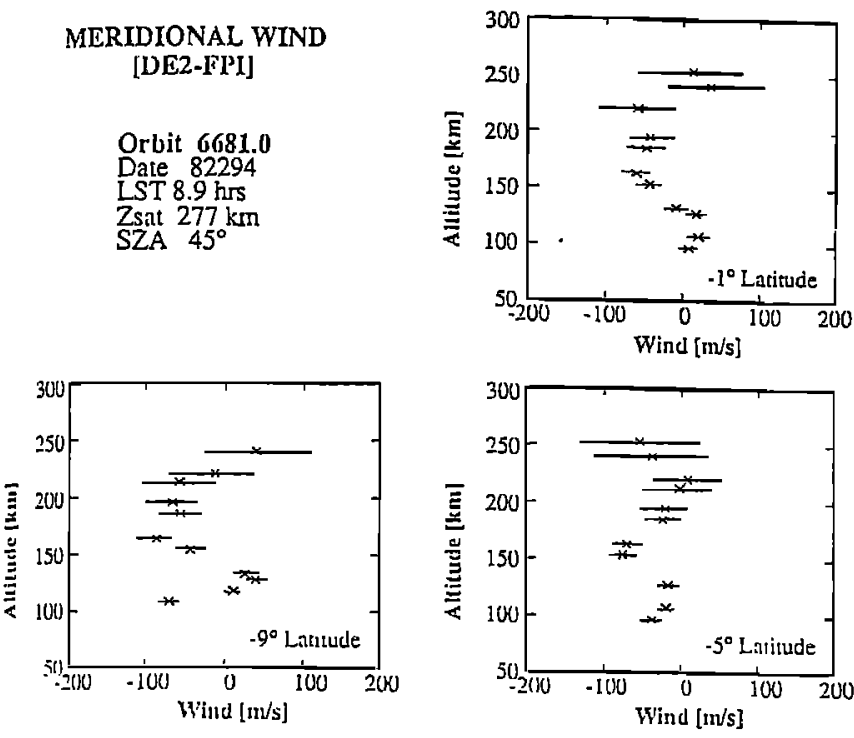

Fig. 3. Examples of dayside lower-thermospheric meridional wind profiles. Errors shown include the effects of the counting statistics and the wind inversion.

07:00 local time plane while, in the second period, the orbit had precessed to the 14:00-2:00 local time plane. Due to the relatively large field of view at the limb and the nature of the VER profiles at high-latitude, these averaged measurements are considered to be representative of a mean altitude of $~ 120$ $\mathrm{km}$. The mean altitude ascribed to the binned data is not very sensitive to the presence or absence of aurora within the field of view, since the auroral $\lambda .557 .7 \mathrm{~nm}$ emission peaks in this same altitude region [Cogger et al., 1985]. The singlecomponent (meridional) wind averages from the two datacollection periods provide separate, near-orthogonal components of the vector wind field that may be combined in the geomagnetic coordinate frame to provide limited vector coverage at latitudes greater than $70^{\circ} \mathrm{S}$ magnetic. The averaged geomagnetic-polar-cap vector wind field obtained in this fashion is shown in Figure 4a and is referred to here as the "synthesized" wind field.

This data handling procedure uses single component averages for all geomagnetic conditions, separated by $\sim 2$ months, and necessarily assumes that the seasonal wind variation over this period is minor compared with spatial variations. While this assumption is questionable, the detailed model comparisons (wherein the model predictions were treated in exactly the same way) indicate that the synthesized high-latitude vector wind field provides a reasonable description of the polar vector wind field for this time period.

The synthesized vector wind field, shown in Figure $4 a$, represents the first large-scale experimental characterization of the lower-thermospheric neutral wind pattern deep within the geomagnetic polar cap. Two striking features are evident. First, there is a region of high-speed, equatorially-directed winds in the early-morning sector of the polar cap. These average winds approach $\sim 300 \mathrm{~m} / \mathrm{sec}$ in magnitude. They are consistent in both speed and magnitude with the equatorward winds observed from the Sondrestrom radar at $74.5^{\circ}$ geomagnetic latitude during a period of moderate geomagnetic activity in 1987 [Johnson and Virdi, 1991]. Second, there is evidence of anticyclonic vorticity in the dayside sector of the geomagnetic polar cap, with the wind field rotating from a sunward direction in the dusk sector to an antisunward

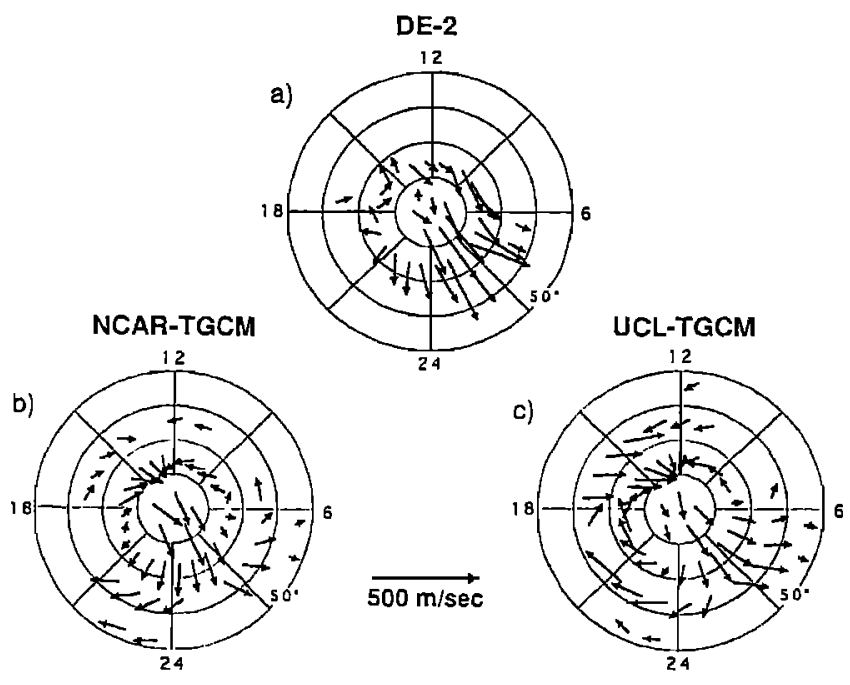

Fig. 4. (a) "Synthesized" winds at $\sim 120 \mathrm{~km}$ over the southern (summer) hemisphere polar region in geomagnetic polar coordinates (outer circle at $50^{\circ} \mathrm{S}$ ). (b) Predicted neutral wind field at $120 \mathrm{~km}$ from the NCAR TGCM. (c) same as (b), except that the UCL model has been used.

direction in the dawn sector. This vorticity resembles that observed at higher altitudes. It is also consistent with incoherent scatter radar observations of lower-thermospheric vorticity reported by Johnson and Virdi [1991]. We interpret both the vorticity and the strong morning-sector equatorial wind evident in our data to be due to the effects of magnetospheric coupling via the ion-drag momentum forcing mechanism. Future investigations will use DE-2 data from the northern hemisphere for which ground-based measurements may be available for comparison

The synthesized experimental wind vectors have been compared with the calculations of two separate TGCMs: the National Center for Atmospheric Research (NCAR) TGCM [Roble et al., 1988] and the University College London (UCL) TGCM [Fuller-Rowell et a1., 1990]. The two models were each run twice for geophysical conditions appropriate to the two DE-2 data sets (solar maximum, moderate geomagnetic activity, November 1982 and January, 1983). The TGCMcalculated meridional winds at the $120 \mathrm{~km}$ altitude level were (vectorially) combined in geomagnetic polar coordinates, in exactly the same manner as was done for the FPI data, to produce the results shown in Figure $4 \mathrm{~b}$ for the NCAR TGCM and Figure $4 c$ for the UCL TGCM. As a test of the validity of this procedure a comparison was made between the two model synthesized vector wind fields and the "normal" vector wind fields calculated for the individual model runs. These comparisons showed that the synthesized theoretical wind field was similar (though not identical in all respects) to the normally-calculated wind field.

The comparison between the TGCM predictions and the $\mathrm{DE}-2$ data indicates a level of agreement that is encouraging. In both cases the models show an early-morning-sector crosspolar wind of $\sim 200 \mathrm{~m} / \mathrm{sec}$ magnitude directed along the 03:00 hrs MLT meridian. The FPI data show an average wind speed of greater magnitude (up to $300 \mathrm{~m} / \mathrm{sec}$ ) in the same direction. The experimental data and the theoretical model results all show the presence of anticyclonic vorticity in the daytime sector, although there are significant discrepancies in both wind magnitude and direction. On the dayside, for example, 
the satellite data indicate a clockwise rotation through noon, while both models show a converging wind field.

\section{Conclusions}

We have presented first lower-thermospheric wind and volume emission rate data from measurements made by the DE-2 FPI. The averaged winds at high geomagnetic latitudes in the summer hemisphere show the presence of significant anticyclonic vorticity in the daytime sector of the polar cap and the presence of a large magnitude $(-300 \mathrm{~m} / \mathrm{sec})$ equatorial wind jet in the early morning sector. These results are consistent with the the ground-based measurements of Johnson and Virdi [1991] in the northern hemisphere. The high-latitude $\mathrm{DE}-2$ results are also in encouraging agreement with the calculations of two thermospheric general circulation models, with significant regional discrepancies.

Acknowledgements. This work was supported by NASA grants NAG5-465, NAGW-1535, and NGT-50193 and by NSF grant ATM-8918476 to the University of Michigan. The authors wish to thank Drs. A. G. Burns, R. M. Johnson, and R. J. Niciejewski for helpful comments.

\section{References}

Cogger, L.L., J.S. Murphree, C.A. Tepley, and J.W. Meriwether Jr., Measurements of the E-region neutral wind field, Planet. Space Sci., 33, 373-379, 1985.

Fuller-Rowell, T.J., D. Rees, B.A. Tinsley, H. Rishbeth, A.S. Rodger, and S. Quegan, Modelling the response of the thermosphere and ionosphere to geomagnetic storms: Effects of a mid-latitude heat source, Adv. Space Res, 10 , 6, 215-224, 1990.

Hays, P.B., T.L. Killeen, and B.C. Kennedy, The FabryPerot interferometer on Dynamics Explorer, Space Sci. Instrum., 5, 395-416, 1981.

Heppner, J.P., and M.L. Miller, Thermospheric winds at high latitudes from chemical release observations, I. Geophys. Res., 87, 1633-1647, 1982.

Hernandez, G., and J.L. Smith, Mesospheric wind determinations and the $\mathrm{P} 1(2) c, d$ lines of the $\mathrm{X}^{2} \mathrm{P} \mathrm{OH}(8-3)$ band, Geophys. Res. Lett. 11, 534-537, 1984.

Johnson, R.M., Lower-thermospheric neutral winds at high latitude determined from incoherent scatter measurements: a review of techniques and observations, Adv. Space Res., 10, 6, 261-275, 1990.

Johnson, R.M., and J.G. Luhmann, High-latitude mesopause neutral winds and geomagnetic activity: A crosscorrelation analysis, J. Geophys. Res., 90, 8501-8506, 1985.

Johnson, R.M., and T.S. Virdi, High-latitude lower thermospheric neutral winds at EISCAT and Sondrestrom during LTCS 1, J. Geophys. Res., 96, A2, 1099-1116, 1991.

Killeen, T.L., and P.B. Hays, Doppler line profile analysis for a multi-channel Fabry-Perot interferometer, Appl. Opt., 23, 612, 1984.

Killeen, T.L., and R.G. Roble, Thermosphere dynamics: Contributions from the first 5 years of the Dynamics Explorer Program, Rev. Geophys., 26, 329-367, 1988.

Lloyd, N., A. H. Manson, D. J. McEwen, and C. E. Meek, A Comparison of Middle Atmospheric Dynamics at Saskatoon $\left(52^{\circ} \mathrm{N}, 107^{\circ} \mathrm{W}\right)$ as Measured by a MediumFrequency Radar and a Fabry-Perot Interferometer, J. Geophys. Res., 95, D6, 7653-7660, 1990.

Manson, A.H., C.E. Meek, S.K. Avery, and D. Tetenbaum, Comparison of mean wind and tidal fields at Saskatoon and Poker Flat during 1983/1984, Phys. Scripta, 37, 169. $177,1988$.

Manson, A.H., C.E. Meek, H. Teitelbaum, F. Vial, R. Schminder, D. Kurschner, M.J. Smith, G.J. Fraser, and R.R. Clark, Climatologies of semidiumal and diurnal tides in the middle atmosphere $(70-110 \mathrm{~km})$ at middle latitudes $\left(40-50^{\circ} \mathrm{N}\right)$, J. Atmos. Terr. Phys., 51, 579-594, 1989.

Nardi, B., An Inversion Technique to Recover Lower Thermospheric Winds from Space-Borne Measurements of [OI] $5577 \AA$., PhD Thesis, University of Michigan, 1991.

Rees, D., A. Aruliah, T.J. Fuller-Rowell, V.B. Wickwar, and R.J. Sica, Winds in the upper mesosphere at midlatitude: First results using an imaging FPI, Geophys. Res. Lett., 17, 1259-1262, 1990.

Roble, R.G., E.C. Ridley, A.D. Richmond, and R.E. Dickinson, A coupled thermosphere/ionosphere general circulation model, Geophys. Res. Lett., 15, 1325-1328, 1988.

Wallace, L., and M. B. McElroy, The Visual Dayglow, Planet. Space Sci., 14, 677, 1966.

Wiens, R.H., G.G. Shepherd, W.A. Gault, and P.R. Kosteniuk, Optical measurements of winds in the lower thermosphere, J. Geophys. Res, 93, 5973-5980, 1988.

T. J. Fuller-Rowell, Space Environmental Research Lab., NOAA, 25 Broadway, Boulder, CO 80303.

T. L. Killeen, B. Nardi, and P. N. Purcell, Space Physics Research Laboratory, The University of Michigan, 2455 Hayward Street, Ann Arbor, MI 48109-2143.

D. Rees, Atmospheric Physics Laboratory, University College London, 67-73 Riding Horse Street, London, U.K. W1P 6PP

R. G. Roble, High Altitude Observatory, National Center for Atmospheric Research, Boulder, CO 80303

(Received: March 11, 1992; accepted: April 3, 1992) 